\title{
Facile Synthesis Strategy from Sludge-Derived Extracellular Polymeric Substances to Nitrogen-Doped Graphene Oxide-Like Material and Quantum Dots
}

\author{
Qiuyuan Lin, Hui Chen,* Jianglin Cao,* and Junxi Zhang
}

Cite This: ACS Omega 2021, 6, 24940-24948

Read Online

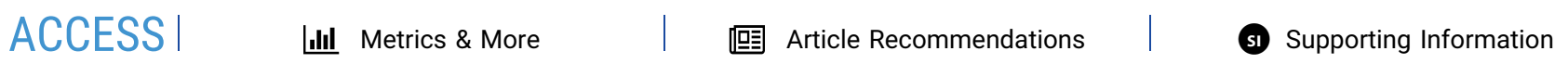

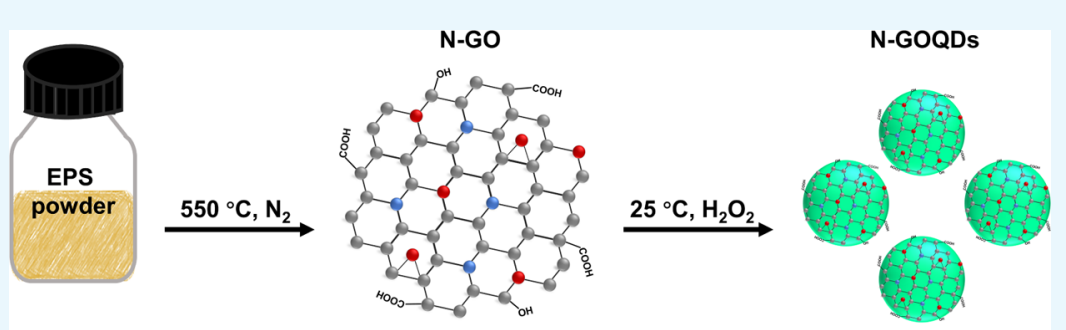

Carbon Oxygen Nitrogen

ABSTRACT: Extracellular polymeric substances (EPS) are microbial aggregates derived from waste sewage sludge accumulated in sewage treatment plants, which provides natural, renewable, and abundant carbon, nitrogen, oxygen sources for the development of carbon materials to achieve the value-added utilization of waste sewage sludge resources. In this work, a nitrogen-doped graphene oxide (GO)-like material (N-GO) was simply produced using EPS as starting materials. A facile $\mathrm{H}_{2} \mathrm{O}_{2}$ oxidation-assisted method (room temperature) was developed to synthesize nitrogen-doped GO-like quantum dots (N-GOQDs) with strong tunable fluorescence from N-GO for the first time. This approach eliminates the conventional use of toxic chemicals, concentrated acids as well as expensive equipment, and strict condition requirements, which provides new insights into the synthesis of $\mathrm{N}-\mathrm{GO}$ and $\mathrm{N}$ GOQDs. In addition, this $\mathrm{H}_{2} \mathrm{O}_{2}$-assisted method was further demonstrated to prepare yellow fluorescent GO quantum dots (GOQDs) from GO successfully. The as-prepared N-GO shows excellent adsorption capacity for removing organic matters (malachite green, rhodamine B, and methylene blue) from water in $10 \mathrm{~min}$. The water-soluble N-GOQDs were demonstrated to be a low toxicity and good biocompatibility fluorescence probe for bioimaging.

\section{INTRODUCTION}

In the past decade, nitrogen-doped graphene ${ }^{1-3}$ and its derivatives such as nitrogen-doped graphene oxide (GO) $)^{4,5}$ and nitrogen-doped GO quantum dots (GOQDs) ${ }^{6,7}$ have attracted extensive attention. Like most graphene materials, nitrogen-doped graphene and its derivatives have similar excellent chemical and physical characters, such as large surface area, low cytotoxicity, excellent biocompatibility, unique optical property, and extraordinary electrical performance. What is more, it has been reported that nitrogen doping can really enhance the properties of graphene such as increasing the electrical conductivity, surface hydrophilicity, adsorption, and catalysis ability. ${ }^{5,8,9}$ The abovementioned characteristics have imparted nitrogen-doped graphene, nitrogen-doped GO, and nitrogen-doped GOQDs a wide range of applications, ranging from photo/electrocatalysis, $4,7,10$ bioimaging, ${ }^{11}$ to biosensing, ${ }^{2}$ fuel cells, and so on.

To date, the preparation of nitrogen-doped GO mostly depends on using GO as a precursor treated with ammonia via a solvothermal process, ${ }^{4}$ hydrothermal method, ${ }^{5}$ or chemical vapor deposition; ${ }^{12}$ these strategies are easy and feasible, but also uneconomical to some extent. The precursor GO is generally prepared by (modified) Hummers' method, ${ }^{13,14}$ which has the risk of explosion and/or burning due to the concentrated acid and strong oxidant treatment. Similarly, various methods have been proposed to prepare graphene quantum dots, ${ }^{15}$ GOQDs, ${ }^{16}$ and nitrogen-doped GOQDs. For example, the "top-down" route involves the acid treatment, addition of nitrogen-containing substances, and chemical exfoliation of graphitic materials of large sizes (e.g., GO, coal, carbon fibers, carbon nanotubes) into small fragments, using modified Hummers' method, laser irradiation, hydrothermal cutting, microwave-assisted cleaving, and so forth. ${ }^{7,17-20}$ These methods slightly suffer from the disadvan-

Received: July 17, 2021

Published: September 20, 2021 
Scheme 1. Schematic Illustration of N-GO and N-GOQDs Synthesis from EPSs ${ }^{a}$

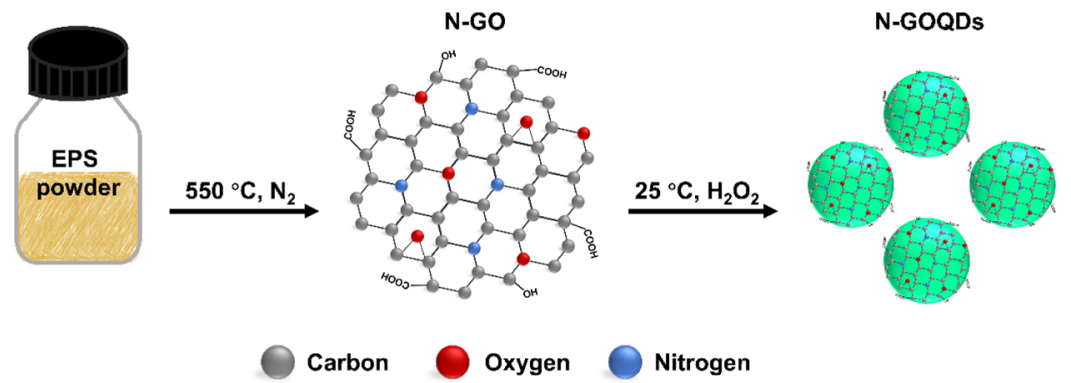

${ }^{a} \mathrm{~N}-\mathrm{GO}, \mathrm{N}-\mathrm{GOQD}$ : nitrogen-doped GO-like quantum dots.
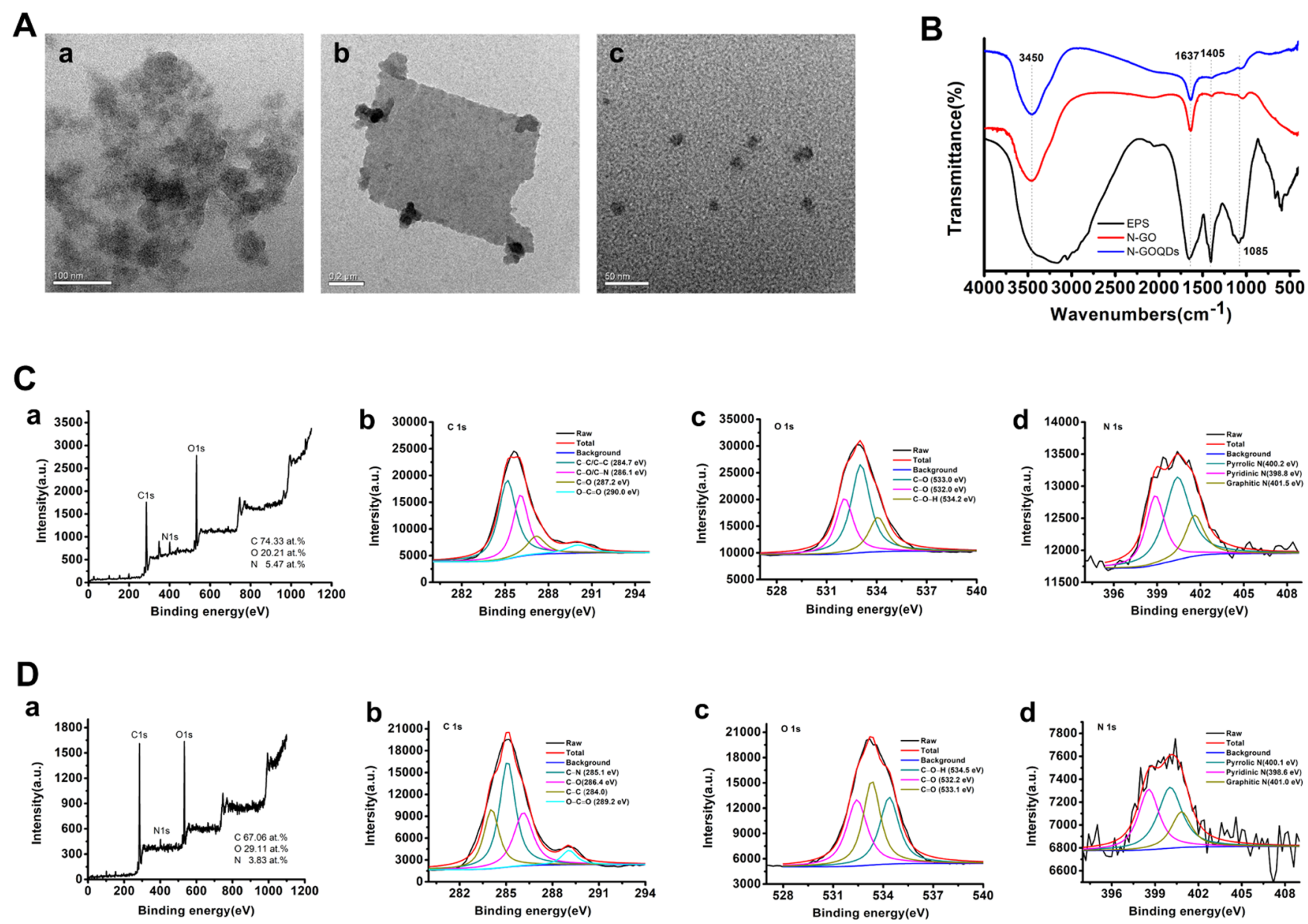

Figure 1. (A) TEM images of EPS (a), N-GO (b), and N-GOQDs (c). FTIR spectra of EPS, N-GO, and N-GOQDs (B). High-resolution XPS spectra with a full range (a), C 1s peak (b), O 1s peak (c), and N 1s (d) of N-GO (C) and N-GOQDs (D).

tages such as requirement of special equipment, pollution of residual chemical agents, and complex synthesis process.

Sludge accumulates as a byproduct during sewage treatment processes in plants. $^{21}$ The continuously increasing quantity of sludge has posed a worldwide environmental problem and thus attracted great attention from environmentalists and chemists. Therefore, it is of great significance as well as an urgent need to utilize sludge as a renewable resource such as exploring approaches to convert useful components in sludge into valuable materials. For example, sludge is currently employed in agriculture as a fertilizer, a source of energy, and so on. ${ }^{22}$ Extracellular polymeric substances (EPS) are one type of the main components present in sludge, primarily composed of high-molecular-weight substances such as proteins, polysaccharides, humic substances, lipids, and nucleic acids, thus making EPS rich in $\mathrm{C}, \mathrm{O}$, and $\mathrm{N}$ elements and excellent precursors for nanomaterial synthesis. ${ }^{23-26}$ Recently, biomass wastes have emerged as a potential precursor for the synthesis of nanomaterials (e.g., carbon quantum dots and graphene quantum dots) generally using hydrothermal treatment, due to their abundant, renewable, cheap, and green characteristics; these prepared nanomaterials exhibited various applications such as metal ion sensing, cell imaging, and supercapacitors. ${ }^{27-34}$ However, EPS derived from waste sewage sludge has not been developed as green and renewable precursors to the synthesis of nitrogen-doped GO and nitrogen-doped GOQD 
A

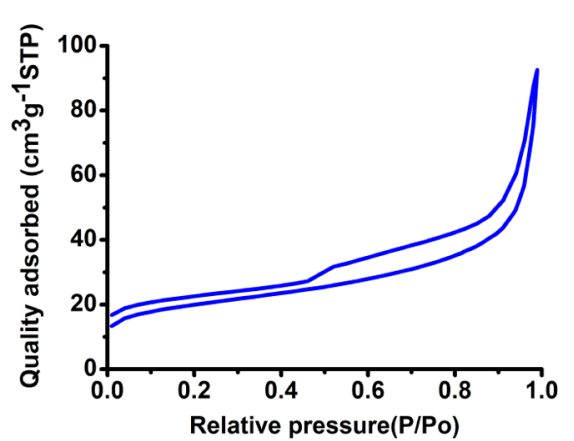

C

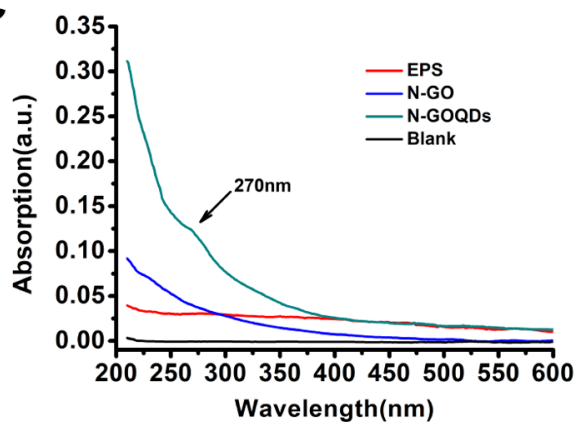

B

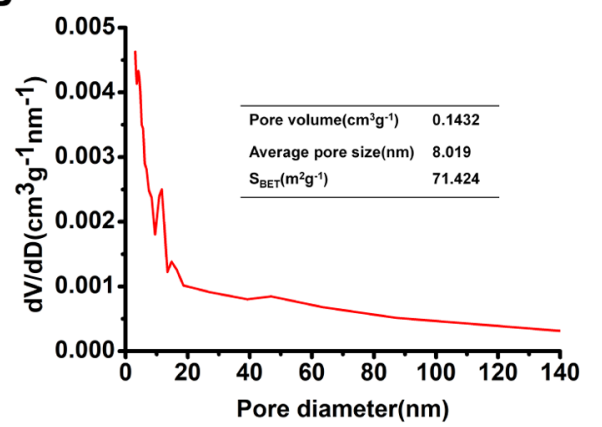

D

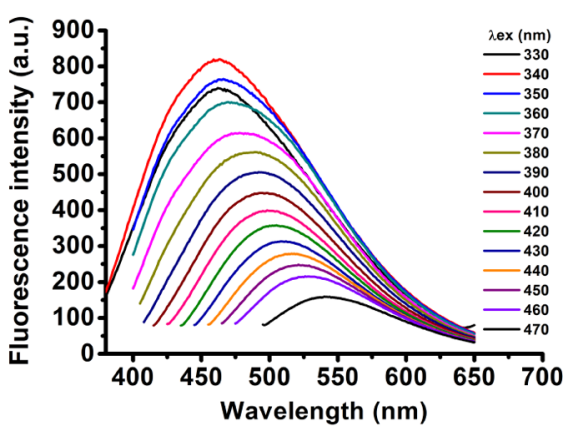

Figure 2. (A) $\mathrm{N}_{2}$ adsorption-desorption curve of N-GO and (B) corresponding pore size distribution. The inset table presents the textural parameters of N-GO. (C) UV-vis absorption of EPS, N-GO, and N-GOQDs in water. (D) Fluorescence emission spectra of N-GQQDs with progressively longer excitation wavelengths $\left(\lambda_{\mathrm{ex}}\right)$ from 330 to $470 \mathrm{~nm}$ in ultrapure water.

analogues, especially using mild methods instead of hydrothermal treatment.

Herein, we report an eco-friendly, nontoxic, low-cost, and easy synthesis method using EPS as the starting materials, which allows to synthesize nitrogen-doped GO-like material (N-GO) and nitrogen-doped GO-like quantum dots (NGOQDs). As illustrated in Scheme 1, EPS powder was utilized as the green $\mathrm{C}, \mathrm{O}$, and $\mathrm{N}$ source materials to produce $\mathrm{N}-\mathrm{GO}$ at $550{ }^{\circ} \mathrm{C}$ for $2 \mathrm{~h}$ without strong acid treatment, which was faster and simpler compared with classic strategies such as solvothermal methods. N-GOQDs with tunable fluorescence emission was synthesized from the as-prepared N-GO using facile $\mathrm{H}_{2} \mathrm{O}_{2}$ oxidation at room temperature. The effectiveness and generality of the $\mathrm{H}_{2} \mathrm{O}_{2}$ oxidation method was further verified by converting $\mathrm{GO}$ into GOQDs with yellow fluorescence emission. N-GO with a large surface area and functional groups exhibited fast and excellent adsorption capacity for toxic organics. Additionally, the water-soluble NGOQDs were successfully applied to cell imaging with good biocompatibility and low toxicity.

\section{RESULTS AND DISCUSSION}

2.1. Synthesis of $\mathrm{N}-\mathrm{GO}$ and N-GOQDs. N-GO was directly synthesized in a tube furnace with a ramp rate of $5{ }^{\circ} \mathrm{C}$ $\min ^{-1}$ to $550^{\circ} \mathrm{C}$ for $2 \mathrm{~h}$ in $\mathrm{N}_{2}$, where EPS acted as the starting source (Scheme 1). The developed synthesis method of N-GO offers many advantages, for example, EPS can be easily extracted from sludge, which is a renewable and green resource and rich in $\mathrm{C}, \mathrm{O}$, and $\mathrm{N}$ elements, thereby acting as an excellent precursor for nanomaterial synthesis. ${ }^{23-26}$ Studies have shown that graphene (oxide) quantum dots (GQDs/ GOQDs) can be synthesized from graphene (oxide); ${ }^{13,35-37}$ however, these synthesis methods mainly rely on strong acid treatment, hydrothermal method, or expensive equipment requirements. It has been reported that hydroxyl radicals $\left({ }^{\bullet} \mathrm{OH}\right)$ dissociated from $\mathrm{H}_{2} \mathrm{O}_{2}$ could facilitate attack to the carbon atoms connected with the hydroxyl and epoxide groups and break carbon-carbon bonds that contribute to generation of GQDs when using the hydrothermal method. ${ }^{38}$ Therefore, we developed a one-step $\mathrm{H}_{2} \mathrm{O}_{2}$-based method for the economical synthesis of N-GOQDs from $\mathrm{N}-\mathrm{GO}$ at mild conditions with a possible mechanism for the formation of $\mathrm{N}$ GOQDs for the first time. Briefly, the layered structure of $\mathrm{N}$ GO was destroyed into small fragments due to the oxidation and exfoliation of $\mathrm{H}_{2} \mathrm{O}_{2}$. With continued processing, the abundant nitrogen and oxygen groups of these small fragments were gradually protonated and became hydroxyl, carboxyl, amino, and so on. Finally, the nano-quantum dot structure of defect-containing N-GOQDs was formed under magnetic stirring (Scheme 1 and Figure 1A). The hydrophilic groups make the N-GOQDs freely dispersed in water, offering good applications, and the facile $\mathrm{H}_{2} \mathrm{O}_{2}$ oxidation-assisted method (room temperature) provides a new strategy for the synthesis of N-GOQDs.

To obtain the N-GOQDs with strong fluorescence, key reaction conditions were optimized, such as the ratio between $\mathrm{N}-\mathrm{GO}$ and $\mathrm{H}_{2} \mathrm{O}_{2}$, reaction time, and temperature for synthesizing $\mathrm{N}-\mathrm{GOQDs}$ by $\mathrm{H}_{2} \mathrm{O}_{2}$ oxidation (Figure $\mathrm{S} 1$ ). As shown in Figure S1A, a noticeable increase of fluorescence intensity is observed when the addition of N-GO rises from 2 $\mathrm{mg}$ to $5 \mathrm{mg}$ in $10 \mathrm{~mL} \mathrm{H}_{2} \mathrm{O}_{2}$ solution; the reaction of $6 \mathrm{mg} \mathrm{N}$ $\mathrm{GO}$ obtained a weaker fluorescence intensity than $5 \mathrm{mg} \mathrm{N}-\mathrm{GO}$, which may be due to the fluorescence quenching by excess $\mathrm{N}$ GO. Meanwhile, the color of N-GOQDs solution also changes from light gold to gold under ambient light, which means that an increase amount of N-GOQDs was produced. As the reaction time increases, the fluorescence intensity of $\mathrm{N}$ - 
A

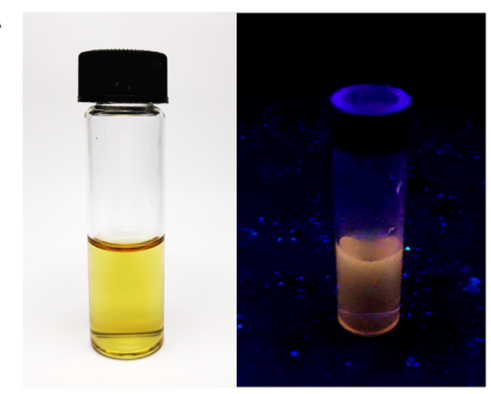

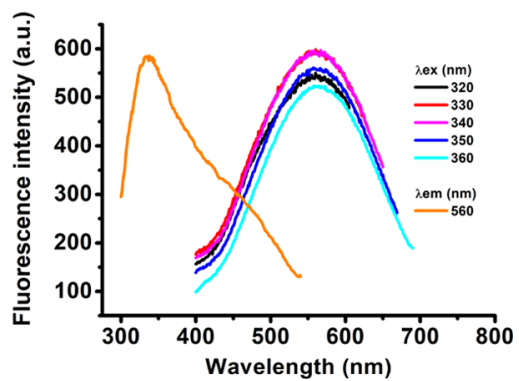

Figure 3. (A) Photographs of the as-prepared GOQDs under ambient light (left) and $365 \mathrm{~nm}$ UV irradiation (right). (B) Fluorescence emission spectra and excitation spectrum of the GOQDs $\left(0.33 \mathrm{mg} \mathrm{mL}^{-1}\right)$ in ultrapure water with $\lambda$ ex from $320 \mathrm{~nm}$ to $360 \mathrm{~nm}$ and $\lambda_{\mathrm{em}}$ at $560 \mathrm{~nm}$. (Photographs were taken by first author: Qiuyuan Lin.)

GOQDs increases significantly, until $56 \mathrm{~h}$, when the reaction reaches equilibrium (Figure S1B). Furthermore, the fluorescence intensity decreases as temperature increases from room temperature $\left(25{ }^{\circ} \mathrm{C}\right.$ ) (Figure S1C). Figure S1D-F illustrates the maximum fluorescence intensity of N-GOQDs prepared under different ratios, reaction times, and temperatures, respectively. In conclusion, an optimized reaction condition: the ratio of $5 \mathrm{mg} / 10 \mathrm{~mL}\left(\mathrm{~N}-\mathrm{GO} / \mathrm{H}_{2} \mathrm{O}_{2}\right), 56 \mathrm{~h}$ reaction time, room temperature $\left(25{ }^{\circ} \mathrm{C}\right)$ was used for preparing N-GOQDs.

2.2. Characterization of EPS, N-GO, and N-GOQDs. The morphology and surface structures of the as-prepared products were first characterized by transmission electron microscopy (TEM). Figure 1Aa illustrates the TEM images of EPS extracted from sludge using a steaming extraction method. EPS are the natural high-molecular-weight polymers produced from microorganisms. EPS comprise a mixture of polysaccharides, proteins, lipids, and nucleic acids (RNA and extracellular DNA) with various functional moieties such as carboxyl, phosphoryl, amide, hydroxyl, amino groups, and so forth. $^{24,25}$ Figure $1 \mathrm{Ab}$ reveals that the as-prepared N-GO had a sheet structure $(\sim \mu \mathrm{m})$. Figure 1Ac shows that N-GOQDs were monodisperse nanoparticles with a size of about $20 \mathrm{~nm}$. Fourier-transform infrared (FTIR) spectra of EPS, N-GO, and $\mathrm{N}-G O Q D s$ are depicted in Figure $1 \mathrm{~B}$; the strong bands at $\sim 3450$ and $\sim 1405 \mathrm{~cm}^{-1}$ were attributed to $\mathrm{O}-\mathrm{H} / \mathrm{N}-\mathrm{H}$ stretching vibrations. ${ }^{6}$ The $\mathrm{C}=\mathrm{C} / \mathrm{C}=\mathrm{N} / \mathrm{C}=\mathrm{O}$ bonds were observed in $\sim 1637 \mathrm{~cm}^{-1}$. ${ }^{39,40}$ The peaks of $\sim 1085 \mathrm{~cm}^{-1}$ indicate the stretching vibration of the $\mathrm{C}-\mathrm{O}$ bond. ${ }^{11,18,41}$ The further X-ray photoelectron spectroscopy (XPS) analysis suggested that the as-prepared products are mainly composed of carbon, oxygen, and nitrogen atoms (Figure 1C,D); Si $(\sim 100 \mathrm{eV})$ and $\mathrm{Ca}(\sim 350 \mathrm{eV})$ elements from the sludge are also found in Figure 1Ca because the isolated EPS (precursor) still contained a small amount of impurities from the sludge. In high-resolution spectra [Figure $1(\mathrm{Cb}),(\mathrm{Db})$ ], the C 1s XPS spectra of $\mathrm{N}-\mathrm{GO}$ and $\mathrm{N}-\mathrm{GOQDs}$ showed the peaks at high binding energy $(285-290 \mathrm{eV})$ due to the presence of the C$\mathrm{C} / \mathrm{C}=\mathrm{C}$ bond $(284.0-284.7 \mathrm{eV}), \mathrm{C}-\mathrm{N}$ bond $(285.1-286.1$ $\mathrm{eV})$, and O-rich groups, such as $\mathrm{C}-\mathrm{O}(286.1-286.4 \mathrm{eV}), \mathrm{C}=$ $\mathrm{O}(287.2 \mathrm{eV})$, and $\mathrm{O}-\mathrm{C}=\mathrm{O}(289.2-290.0 \mathrm{eV}) .^{5,6,10,11}$ The high-resolution $\mathrm{O} 1 \mathrm{~s}$ peak [Figure $1(\mathrm{Cc}),(\mathrm{Dc})$ ] manifests that peaks at about 532.0, 533.0, and $534.2 \mathrm{eV}$ are attributed to the presence of $\mathrm{C}-\mathrm{O}, \mathrm{C}=\mathrm{O}$, and $\mathrm{C}-\mathrm{O}-\mathrm{H}$ groups. ${ }^{42}$ The highresolution $\mathrm{N} 1 \mathrm{~s}$ spectra of $\mathrm{N}-\mathrm{GO}$ and $\mathrm{N}-\mathrm{GOQDs}$ [Figure $1(\mathrm{Cd}),(\mathrm{Dd})]$ revealed the presence of nitrogen species: pyridine-like (398.6-398.8 eV), pyrrolic (400.1-400.2 eV), and graphitic $\mathrm{N}$ atoms $(401.0-401.5 \mathrm{eV}) .^{6,40}$ The contents of
C, O, and $\mathrm{N}$ elements in N-GO are 74.33, 20.21, and 5.47\%, respectively, and those in N-GOQDs are 67.06, 29.11, and $3.83 \%$ respectively. These results together demonstrated the successful synthesis of N-GO and N-GOQDs from EPS.

2.3. Textural and Optical Properties. The textural property of $\mathrm{N}-\mathrm{GO}$ was characterized by $\mathrm{N}_{2}$ adsorption and desorption measurements. The $\mathrm{N}_{2}$ adsorption/desorption isotherms of $\mathrm{N}-\mathrm{GO}$ present a type-IV isotherm as the classification of the International Union of Pure and Applied Chemistry (Figure 2A). ${ }^{43-45}$ The surface area calculated from the Brunauer-Emmett-Teller (BET) curve indicates a surface area of $71.424 \mathrm{~m}^{2} \mathrm{~g}^{-1}$ and pore volume of $0.14 \mathrm{~cm}^{3} \mathrm{~g}^{-1}$ (Figure 2B), which is similar to the reported values of GO. ${ }^{46} \mathrm{~N}-\mathrm{GO}$ exhibits an average pore width of $8.02 \mathrm{~nm}$, indicating that $\mathrm{N}$ GO allows for the adsorption of accessible molecules onto the surface and into the pore.

The UV-vis absorption spectrum of N-GOQDs displays an absorption band at $270 \mathrm{~nm}$ corresponding to the $n-\pi^{*}$ electronic transitions of a polyaromatic structure (Figure 2C), which is consistent with previous reports, ${ }^{6,11}$ while EPS and NGO have no obvious absorption. Figure $2 \mathrm{D}$ shows that the fluorescence emission peak of N-GOQDs gradually red-shifts from 460 to $540 \mathrm{~nm}$ with the excitation wavelength increasing from 330 to $470 \mathrm{~nm}$, and the maximum emission is observed at the wavelength of $460 \mathrm{~nm}$. It can be concluded that N-GOQDs have a noticeable excitation-dependent fluorescence emission property, suggesting that surface defects might be involved in the photoluminescence process due to the porous structure of $\mathrm{N}-\mathrm{GO}$ and the oxidation result of $\mathrm{H}_{2} \mathrm{O}_{2}$. The exact photoluminescence mechanism of N-GOQDs may be the result of doping, electron-hole recombination, quantum effect, edge structure, and surface defects in the functional groups of N-GOQDs according to previous reports. ${ }^{6,11}$ Moreover, the fluorescence quantum yield (QY) of the N-GOQDs was calculated to be $11.2 \%$. The fluorescence behavior of the $\mathrm{N}$ GOQDs was found to be $\mathrm{pH}$-dependent, and the maximum intensity of the emission spectra decreases as the $\mathrm{pH}$ increases from 3.0 to 11.0 (Figure S2). The $\mathrm{pH}$-dependent fluorescence property of the $\mathrm{N}$-GOQDs may be that the increasing concentration of $\mathrm{OH}^{-}$ions induce the significant change of functional groups, and too much negative charge carried by $\mathrm{N}$ GOQDs might inhibit the excited states of the N-GOQDs. Moreover, the N-GOQDs still maintain relatively strong fluorescence intensity over the $\mathrm{pH}$ range 3.0-7.0, suggesting that the $\mathrm{N}-\mathrm{GOQDs}$ can be used in a relative wide $\mathrm{pH}$ range.

2.4. Applying $\mathrm{H}_{2} \mathrm{O}_{2}$-Based Method to the Synthesis of GOQDs. The generality of this developed $\mathrm{H}_{2} \mathrm{O}_{2}$ oxidationassisted method was further verified by preparing GOQDs. 2 

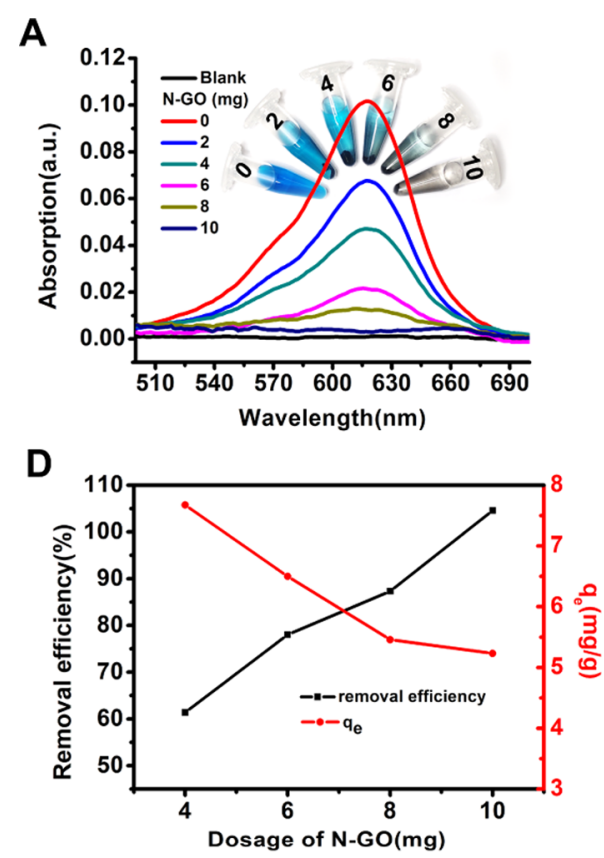
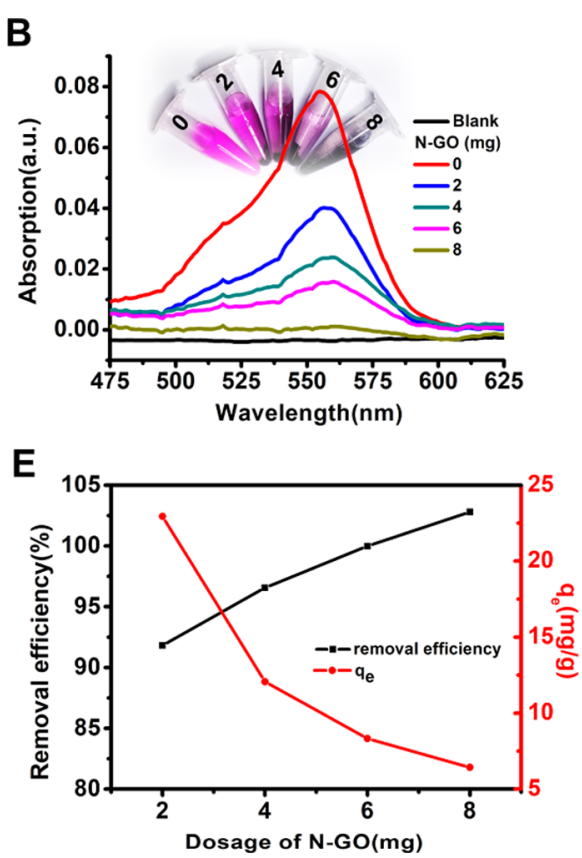

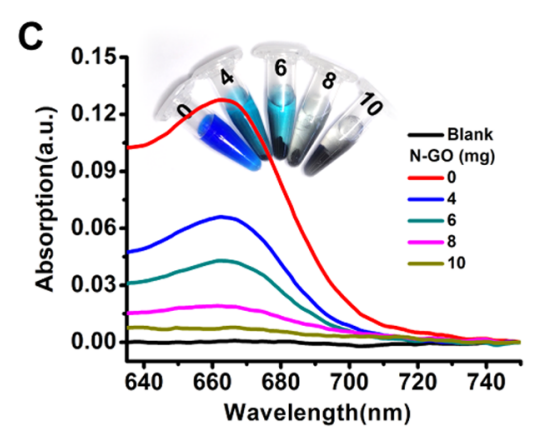

$\mathbf{F}$

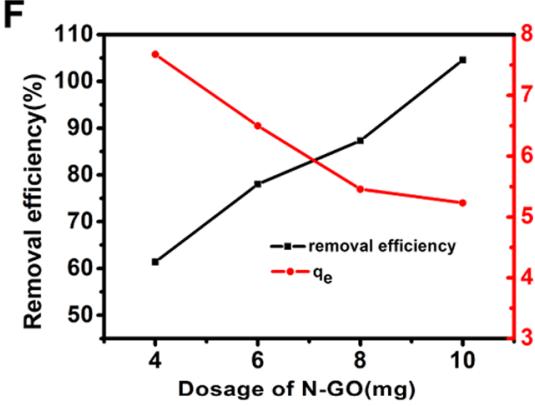

Figure 4. Absorption spectra of $1 \mathrm{~mL}$ of $50 \mathrm{mg} \mathrm{L}^{-1} \mathrm{MG}(\mathrm{A}), \mathrm{RB}(\mathrm{B})$, and $\mathrm{MB}$ (C) with increasing dosage of N-GO. The corresponding effect of the dosage of N-GO powder on the removal efficiency of $\mathrm{MG}(\mathrm{D}), \mathrm{RB}(\mathrm{E})$, and $\mathrm{MB}(\mathrm{F})$ (temperature $25^{\circ} \mathrm{C}, \mathrm{pH}=7$ and reaction time $10 \mathrm{~min}$, Black line). The adsorption capacity $\left(q_{\mathrm{e}}\right)$ of the increasing dosage of $\mathrm{N}-\mathrm{GO}$ for adsorption of MG (D), RB (E), and MB (F) (red line). The inset picture in $(\mathrm{A}-\mathrm{C})$ presents a practical adsorption result. Blank, ultrapure water. (Photographs were taken by first author: Qiuyuan Lin.)

$\mathrm{mL}$ of $\mathrm{GO}$ aqueous solution $\left(2 \mathrm{mg} \mathrm{mL}^{-1}\right)$ was mixed with 10 $\mathrm{mL}$ of $\mathrm{H}_{2} \mathrm{O}_{2}$ and reacted for $60 \mathrm{~h}$ with stirring at room temperature. Surprisingly, yellow emission was obtained from the final product, GOQDs (Figure 3A). As depicted in Figure $3 \mathrm{~B}$, the fluorescence peaks of GOQDs are located at $560 \mathrm{~nm}$ and has a maximum excitation peak at $330 \mathrm{~nm}$. It was also observed that GOQDs exhibited excitation-independent fluorescence characteristic, which is different from $\mathrm{N}$ GOQDs. Compared to GOQDs prepared at high temperature, ${ }^{13}$ GOQDs prepared in this study show a red shift with yellow emission, indicating that the reaction temperature can affect the emission properties of as-synthesized GOQDs and that lower temperature leads to a red shift, which is consistent with the results of a previous study. ${ }^{18}$ Also, the oxidation of $\mathrm{H}_{2} \mathrm{O}_{2}$ has a significant effect on the fluorescence generation of GOQDs and N-GOQDs. Besides, the emission spectra of NGOQDs has an obvious blue shift compared to GOQDs; this result could be attributed to the nitrogen-containing groups, oxygen-rich groups, and the size and surface effects. ${ }^{6}$ Therefore, this result proved that the $\mathrm{H}_{2} \mathrm{O}_{2}$ oxidation method provided a green, facile, and simple way to synthesize QDs universally.

2.5. Adsorption Capacity of N-GO. The nitrogen doping has been reported to create more active sites and enhance many characteristics of graphene materials such as electrochemical catalytic activity, hydrophilicity, and the adsorption capacity because of the greater electronegativity of nitrogen than that of carbon, which offers a great number of applications. ${ }^{3,5,9,47}$ For example, studies found that GO with nitrogen doping greatly enhanced the adsorption ability for removing bisphenols ${ }^{5}$ and boron ions. ${ }^{48}$ The adsorption capacity was first increased and then decreased with the increase of nitrogen doping, and when the atomic ratio of N/C was between 0.062 and 0.073 , nitrogen-doped GO reached its strongest adsorption ability. ${ }^{48} \mathrm{Fe}_{3} \mathrm{O}_{4}$ nanoparticle modification was demonstrated to make GO an attractive adsorbent with enhanced adsorption ability. ${ }^{49}$ Sulfur doping also contributes to improving the adsorption ability of GO for removal of cesium ions. ${ }^{50}$ Moreover, a large surface area with a porous structure of the prepared N-GO was determined by BET, which was reported to play an important role in the adsorption ability of nanomaterials. ${ }^{51}$ Based on this, we test the adsorption capacity of N-GO using malachite green (MG), rhodamine B $(\mathrm{RB})$, and methylene blue (MB) as adsorption targets. $50 \mathrm{mg} /$ $\mathrm{L}$ of $\mathrm{MG}, \mathrm{RB}$, and $\mathrm{MB}$ was mixed with different amounts of $\mathrm{N}$ GO powder and stirred for $10 \mathrm{~min}$ at room temperature. After centrifugation, the absorption curves of these supernatants were detected by a UV-vis spectrophotometer. Figure $4 \mathrm{~A}-\mathrm{C}$ presents the adsorption of $M G, R B$, and $M B$ changed with an increasing addition of $\mathrm{N}-\mathrm{GO}$, respectively. We can observe that these solutions became colorless. The variations of the corresponding maximum absorption values and the final equilibrium concentration of $\mathrm{MG}, \mathrm{RB}$, and $\mathrm{MB}$ are listed in Tables $S 1-S 3$ respectively. The removal efficiency of MG, RB, and $\mathrm{MB}$ was calculated according to the following equation: removal efficiency $\left(R_{\mathrm{e}}\right)=\left(C_{0}-C_{\mathrm{e}}\right) / C_{0} \times 100 \%$, ${ }^{52}$ where $C_{0}$ $(\mathrm{mg} / \mathrm{L})$ is the initial concentration of $\mathrm{MG}, \mathrm{RB}$, and $\mathrm{MB}$ (50 $\mathrm{mg} / \mathrm{L}), C_{\mathrm{e}}(\mathrm{mg} / \mathrm{L})$ is the equilibrium concentration of $\mathrm{MG}$, $\mathrm{RB}$, and $\mathrm{MB}$ after the addition of N-GO. $C_{\mathrm{e}}$ was calculated from the established standard curve representing the relationship between the concentration of $\mathrm{MG}, \mathrm{RB}$, and $\mathrm{MB}$ and absorption (Figure S3). The results in Figure 4D-F show that the removal efficiency of MG, RB, and MB sharply increased to about $100 \%$ as the dosage of N-GO increased, due to the abundant active adsorption sites and large surface area of $\mathrm{N}$ GO. The adsorption capacity $q_{\mathrm{e}}(\mathrm{mg} / \mathrm{g})$ was determined as: $q_{\mathrm{e}}$ $=\left(C_{0}-C_{\mathrm{e}}\right) \times \mathrm{V} / \mathrm{m}$ according to previous reports. ${ }^{52,53} \mathrm{~V}(\mathrm{~L})$ is the total volume of the solution, $\mathrm{m}(\mathrm{g})$ is the dosage of $\mathrm{N}-\mathrm{GO}$, and $\mathrm{q}_{\mathrm{e}}(\mathrm{mg} / \mathrm{g}$ ) is the amount of MG (or RB or MB) adsorbed per unit weight of N-GO. The adsorption capacity $\left(q_{e}\right)$ of the 
$\mathrm{N}-\mathrm{GO}$ was $9.78,22.95$, and $7.68 \mathrm{mg} / \mathrm{g}$ when the dosage for removing $M G, R B$, and $M B$ was 2,2 , and $4 \mathrm{~g} / \mathrm{L}$, respectively. The results showed that the prepared N-GO (atomic ratio of $\mathrm{N} / \mathrm{C}$ : 0.074) efficiently removed $M G, R B$, and $M B$ from aqueous solutions in $10 \mathrm{~min}$, which were highly consistent with previous report. ${ }^{48}$ Similar to biomass-based adsorbents, ${ }^{54,55}$ we think that many other factors such as electrostatic interactions, $\pi-\pi$ stacking, and hydrogen bonding also contribute to the adsorption efficiency of sludge-derived N-GO, which shows a great potential in toxic organic chemical removal from contaminated water.

2.6. Cellular Imaging. The toxicity of the as-prepared $\mathrm{N}$ GOQDs was evaluated by the 3-(4,5-dimethylthiazol-2-yl)-2,5diphenyltetrazolium bromide (MTT) assay before exploring bioimaging applications. SGC 7901 and LoVo cells were selected as models in this study. As shown in Figure 5, the

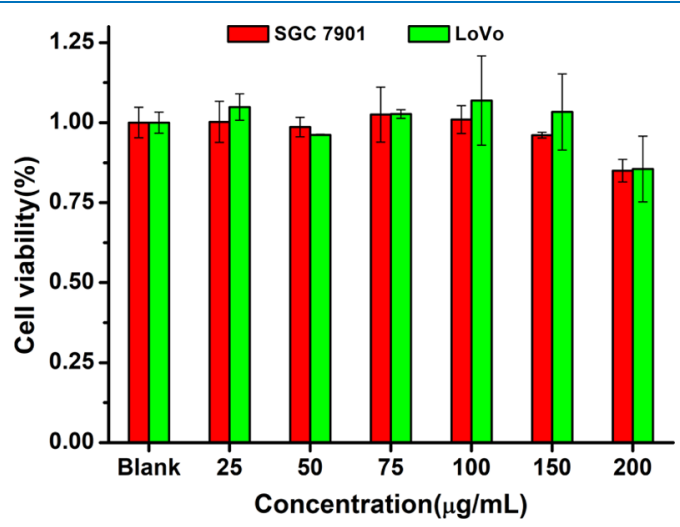

Figure 5. Cell viability assay of SGC 7901 and LoVo cells in the presence of different concentrations of the N-GOQDs.

cellular viability of both SGC 7901 and LoVo cells remains approximately $85 \%$ even with the concentration of the $\mathrm{N}$ GOQDs up to $200 \mu \mathrm{g} \mathrm{mL}^{-1}$. The result suggested that the $\mathrm{N}$ GOQDs had low toxicity and good biocompatibility, which could be further used for cellular imaging. Then, SGC 7901 and LoVo cells were cultured for $4 \mathrm{~h}$ at $37{ }^{\circ} \mathrm{C}$ in $150 \mu \mathrm{g} \mathrm{mL}^{-1}$ $\mathrm{N}-G O Q D s$ and subsequently analyzed under a fluorescence microscope with the excitation of $488 \mathrm{~nm}$. Figure 6 shows that both SGC 7901 and LoVo cells were stained by the fluorescence of N-GOQDs and exhibited a clear morphology with green emission under a $488 \mathrm{~nm}$ excitation channel, suggesting that the synthesized N-GOQDs act as a biocompatible and economical probe for cell bioimaging and labeling applications.

\section{CONCLUSIONS}

In summary, a facile synthesis method is reported to synthesize $\mathrm{N}-\mathrm{GO}$ and N-GOQDs with strong tunable fluorescence emission from EPS for the first time. We proved that the EPS-derived N-GO can serve as an excellent adsorbent for removing organic pollutants in water with high efficiency. The room temperature $\mathrm{H}_{2} \mathrm{O}_{2}$ oxidation strategy was developed to prepare N-GOQDs and GOQDs, which show wide application potential because of their advantages of being clean, easily available, and low-cost. N-GOQDs were found to be low in cytotoxicity and had excellent biocompatibility and were used for cell imaging.
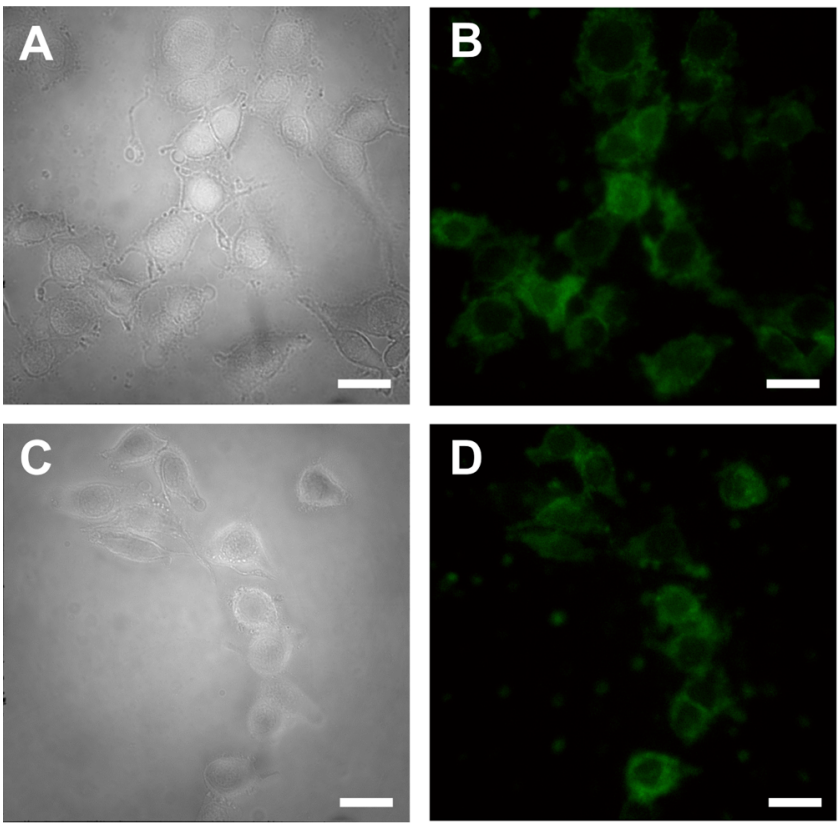

Figure 6. Bright field image (A), fluorescence image under the excitation of $488 \mathrm{~nm}$ (B) of SGC 7901 cells incubated with $\mathrm{N}$ GOQDs for $12 \mathrm{~h}$. Bright field image (C), fluorescence image under the excitation of $488 \mathrm{~nm}$ (D) of LoVo cells incubated with N-GOQDs for $4 \mathrm{~h}$. Scale bar, $20 \mu \mathrm{m}$.

\section{EXPERIMENTAL SECTION}

4.1. Reagents and Instrumentation. Sludge was obtained from a sewage treatment plant in Shanghai, China. Dulbecco's modified Eagle's media (DMEM), fetal bovine serum (FBS), and phosphate buffered saline (PBS, pH 7.0) were purchased from Solarbio Science \& Technology Co., Ltd (Beijing, China). An MTT cell proliferation assay kit was purchased from Multi Sciences(lianke) Biotech, Co., Ltd (Hangzhou, China). The human cell lines, SGC 7901 cells, and LoVo cells were obtained from Chinese Academy of Sciences Cell bank (Shanghai, China). MG, RB, MB, hydrochloric acid $(\mathrm{HCl})$, sodium hydroxide $(\mathrm{NaOH})$, quinine sulfate, $\mathrm{H}_{2} \mathrm{SO}_{4}$, and hydrogen peroxide $\left(30 \% \mathrm{H}_{2} \mathrm{O}_{2}\right)$ were obtained from China National Pharmaceutical Group Co., Ltd. (Sinopharm) (Beijing, China). GO $\left(2 \mathrm{mg} \mathrm{mL}^{-1}\right.$, dispersion in $\mathrm{H}_{2} \mathrm{O}$ ) was obtained from Sigma-Aldrich. All chemicals were used without further purification. Ultrapure water (18 $\mathrm{M} \Omega$ $\mathrm{cm}^{-1}$ ) was obtained from a Milli-Q gradient system for all experiments.

UV-visible spectra were taken in a SMA4000 UV-vis spectrophotometer. The fluorescence spectra were recorded using an Agilent Cary Eclpse fluorescence spectrophotometer (Agilent Technologies, USA). The size and morphology studies of the as-prepared samples were performed by TEM (JEM 2011) at an accelerating voltage of $120 \mathrm{kV}$. XPS asprepared samples were recorded on a PHI 5000C\&PHI5300 spectrometer. The contents of elements of the prepared materials were determined by a Vario EL elemental analyzer (Analysemsysteme GmbH, Langenselbold, Germany). Fourier transform infrared (FT-IR) spectra were recorded on a Thermo Scientific Nicolet iS10 FT-IR spectrometer using $\mathrm{KBr}$ pellets. The BET surface areas of the products were acquired with Quadrasorb evo full-automatic adsorption analyzer. The QY of the N-GOQDs was measured according to a previous report. ${ }^{56}$ The absorption intensity was obtained 
on a SMA4000 UV-vis spectrophotometer. Quinine sulfate in $0.1 \mathrm{M}$ of $\mathrm{H}_{2} \mathrm{SO}_{4}$ that has a standard fluorescence $\mathrm{QY}$ value $(54 \%$ at $360 \mathrm{~nm})$ was chosen as a reference sample. The adsorption isotherms of $\mathrm{MG}$ by $\mathrm{N}-\mathrm{GO}$ were performed referring to a previous study. ${ }^{52}$ The concentration of MG, $\mathrm{RB}$, and $\mathrm{MB}$ in the aqueous solutions was determined via a UV spectrophotometer. Fluorescence imaging was performed with an inverted fluorescence microscope (Nikon Eclipse Ti Series).

4.2. Extraction of EPS from Sludge. The steaming extraction method proposed previously was used for extracting EPS with some modification. ${ }^{57} 200 \mathrm{~g}$ of sludge was dissolved in $1000 \mathrm{~mL}$ of ultrapure water with stirring. Then, the sludge solution was steamed in an autoclave for $15 \mathrm{~min}$ and then centrifuged at $10,000 \mathrm{rpm} / \mathrm{min}$ for $10 \mathrm{~min}$. The obtained supernatants were filtered through $0.22 \mu \mathrm{m}$ cellulose acetate filters to soluble fractions. EPS were dried in an oven at $50{ }^{\circ} \mathrm{C}$ and ground into powder. EPS powder was sealed and stored at $4{ }^{\circ} \mathrm{C}$.

4.3. Synthesis of N-GO from EPS. $1.0 \mathrm{~g}$ of EPS powder was heated in a tube furnace with a ramp rate of $5{ }^{\circ} \mathrm{C} \mathrm{min}^{-1}$ to $550{ }^{\circ} \mathrm{C}$ for $2 \mathrm{~h}$ in $\mathrm{N}_{2}$ to obtain $\mathrm{N}-\mathrm{GO}$ by referring to previous reports with some modifications. ${ }^{58-60}$ After the reaction, the solid precipitates were ground into powder and dispersed in 6 $\mathrm{M} \mathrm{HCl}$ solution to remove metal impurities. ${ }^{61}$ Then, the asprepared N-GO was washed with ultrapure water several times, followed by drying at $60{ }^{\circ} \mathrm{C}$.

4.4. Synthesis of N-GOQDs. N-GOQDs was one-step synthesized from $\mathrm{N}-\mathrm{GO}$ with $\mathrm{H}_{2} \mathrm{O}_{2}$ treatment. In brief, $0.005 \mathrm{~g}$ of a N-GO-like material was dispersed in $10 \mathrm{~mL}$ of $\mathrm{H}_{2} \mathrm{O}_{2}$ aqueous solution through magnetic stirring at room temperature for $12-56 \mathrm{~h}$. Then, the light yellow solution was heated to decompose excess $\mathrm{H}_{2} \mathrm{O}_{2}$. The light yellow solution was dialyzed using a dialysis bag $(3500 \mathrm{Da})$ soaked in ultrapure water for 2 days to remove impurities. After dialysis, the aqueous dispersion was centrifuged at $10,000 \mathrm{rpm} / \mathrm{min}$ to remove any agglomerates. Solid N-GOQDs was obtained by drying at $60{ }^{\circ} \mathrm{C}$.

4.5. MTT Assay for the Cell Cytotoxicity. Both SGC 7901 and LoVo cells were cultured in DMEM medium with $10 \%(\mathrm{v} / \mathrm{v}) \mathrm{FBS}$ and $1 \%$ penicillin/streptomycin at $37{ }^{\circ} \mathrm{C}$ in a humidified $5 \% \mathrm{CO}_{2}$ atmosphere. For the MTT assay, $100 \mu \mathrm{L}$ of SGC 7901 and LoVo cells were seeded into a 96-well plate. After a $24 \mathrm{~h}$ interval, the culture medium was discarded and the cells were treated with $100 \mu \mathrm{L}$ fresh DMEM containing various concentrations of N-GOQDs $\left(0-200 \mu \mathrm{g} \mathrm{mL}^{-1}\right)$ for 24 h. Then, $10.0 \mu \mathrm{L}$ of MTT ( $5 \mathrm{mg} \mathrm{mL}^{-1}$ ) was added to each well for $4 \mathrm{~h}$. Finally, the culture medium was discarded and the cells were cured with $100 \mu \mathrm{L}$ formazan solvent. The optical density of solubilized formazan salts was evaluated at $570 \mathrm{~nm}$ by an automatic microplate reader (Thermo Scientific).

4.6. Cellular Imaging. The potential for cellular imaging of the obtained N-GOQDs was tested by SGC 7901 and LoVo cells. SGC 7901 and LoVo cells were incubated with NGOQDs $\left(150 \mu \mathrm{g} \mathrm{mL}^{-1}\right)$ at $37{ }^{\circ} \mathrm{C}$ for $12 \mathrm{~h}$ and washed three times by PBS solution. Fluorescent images of cells were obtained using a Nikon Eclipse Ti Series microscope with the $\lambda$ ex $488 \mathrm{~nm}$.

\section{ASSOCIATED CONTENT}

\section{SI Supporting Information}

The Supporting Information is available free of charge at https://pubs.acs.org/doi/10.1021/acsomega.1c03804.
Optimization of the synthesis condition; fluorescence emission spectra of N-GQQDs in the different $\mathrm{pH}$ ranging from 3.0 to 11.0; linear relationship between the maximum absorption value and the concentration of $\mathrm{MG}, \mathrm{RB}$, and $\mathrm{MB}$, respectively; and maximum absorption value of $\mathrm{MG}, \mathrm{RB}$, and $\mathrm{MB}$ after the increasing addition of $\mathrm{N}-\mathrm{GO}$, respectively (PDF)

\section{AUTHOR INFORMATION}

\section{Corresponding Authors}

Hui Chen - Department of Chemistry, Fudan University,

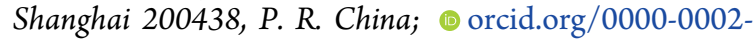
2281-5935; Email: chenhui@fudan.edu.cn

Jianglin Cao - State Key Laboratory of Pollution Control and Resource Reuse, College of Environmental Science and Engineering, Tongji University, Shanghai 200092, P. R. China; Shanghai Institute of Pollution Control and Ecological Security, Shanghai 200092, China; Email: jlcao@

tongji.edu.cn

\section{Authors}

Qiuyuan Lin - Department of Chemistry, Fudan University, Shanghai 200438, P. R. China

Junxi Zhang - Shanghai Institute of Pollution Control and Ecological Security, Shanghai 200092, China; Shanghai Key Laboratory of Materials Protection and Advanced Materials in Electric Power, Shanghai University of Electric Power, Shanghai 200090, P. R. China; 이이이.org/0000-00017055-8892

Complete contact information is available at:

https://pubs.acs.org/10.1021/acsomega.1c03804

\section{Notes}

The authors declare no competing financial interest.

\section{ACKNOWLEDGMENTS}

This work was jointly supported by The Major Science and Technology Program of China for Water Pollution Control and Treatment (grant no. 2017ZX07202), Shanghai Science and Technology Innovation Action Plan (20392001900), Shanghai Natural Science Foundation (20ZR1403000).

\section{REFERENCES}

(1) Duan, X.; Ao, Z.; Sun, H.; Indrawirawan, S.; Wang, Y.; Kang, J.; Liang, F.; Zhu, Z. H.; Wang, S. Nitrogen-doped graphene for generation and evolution of reactive radicals by metal-free catalysis. ACS Appl. Mater. Interfaces 2015, 7, 4169-4178.

(2) Wang, Y.; Shao, Y.; Matson, D. W.; Li, J.; Lin, Y. NitrogenDoped Graphene and Its Application in Electrochemical Biosensing. ACS Nano 2010, 4, 1790-1798.

(3) Kaur, M.; Kaur, M.; Sharma, V. K. Nitrogen-doped graphene and graphene quantum dots: A review onsynthesis and applications in energy, sensors and environment. Adv. Colloid Interface Sci. 2018, 259, 44-64.

(4) Dumont, J. H.; Martinez, U.; Artyushkova, K.; Purdy, G. M.; Dattelbaum, A. M.; Zelenay, P.; Mohite, A.; Atanassov, P.; Gupta, G. Nitrogen-Doped Graphene Oxide Electrocatalysts for the Oxygen Reduction Reaction. ACS Appl. Nano Mater. 2019, 2, 1675-1682.

(5) Wang, X.; Qin, Y.; Zhu, L.; Tang, H. Nitrogen-Doped Reduced Graphene Oxide as a Bifunctional Material for Removing Bisphenols: Synergistic Effect between Adsorption and Catalysis. Environ. Sci. Technol. 2015, 49, 6855-6864. 
(6) Li, Y.; Zhao, Y.; Cheng, H.; Hu, Y.; Shi, G.; Dai, L.; Qu, L. Nitrogen-doped graphene quantum dots with oxygen-rich functional groups. J. Am. Chem. Soc. 2012, 134, 15-18.

(7) Yeh, T.-F.; Teng, C.-Y.; Chen, S.-J.; Teng, H. Nitrogen-doped graphene oxide quantum dots as photocatalysts for overall watersplitting under visible light illumination. Adv. Mater. 2014, 26, 32973303.

(8) Xu, H.; Ma, L.; Jin, Z. Nitrogen-doped graphene: Synthesis, characterizations and energy applications. J. Energy Chem. 2018, 27, 146-160.

(9) Sun, H.; Wang, Y.; Liu, S.; Ge, L.; Wang, L.; Zhu, Z.; Wang, S. Facile synthesis of nitrogen doped reduced graphene oxide as a superior metal-free catalyst for oxidation. Chem. Commun. 2013, 49, 9914-9916.

(10) Zhang, Y.; Fugane, K.; Mori, T.; Niu, L.; Ye, J. Wet chemical synthesis of nitrogen-doped graphene towards oxygen reduction electrocatalysts without high-temperature pyrolysis. J. Mater. Chem. 2012, 22, 6575 .

(11) Chen, W.; Li, D.; Tian, L.; Xiang, W.; Wang, T.; Hu, W.; Hu, Y.; Chen, S.; Chen, J.; Dai, Z. Synthesis of graphene quantum dots from natural polymer starch for cell imaging. Green Chem. 2018, 20, $4438-4442$.

(12) Li, X.; Wang, H.; Robinson, J. T.; Sanchez, H.; Diankov, G.; Dai, H. Simultaneous Nitrogen Doping and Reduction of Graphene Oxide. J. Am. Chem. Soc. 2009, 131, 15939-15944.

(13) Liu, B.; Xie, J.; Ma, H.; Zhang, X.; Pan, Y.; Lv, J.; Ge, H.; Ren, N.; Su, H.; Xie, X.; Huang, L.; Huang, W. From Graphite to Graphene Oxide and Graphene Oxide Quantum Dots. Small 2017, 13, 1601001.

(14) Shi, Y.; Pramanik, A.; Tchounwou, C.; Pedraza, F.; Crouch, R. A.; Chavva, S. R.; Vangara, A.; Sinha, S. S.; Jones, S.; Sardar, D.; Hawker, C.; Ray, P. C. Multifunctional biocompatible graphene oxide quantum dots decorated magnetic nanoplatform for efficient capture and two-photon imaging of rare tumor cells. ACS Appl. Mater. Interfaces 2015, 7, 10935-10943.

(15) Huang, Z.; Shen, Y.; Li, Y.; Zheng, W.; Xue, Y.; Qin, C.; Zhang, B.; Hao, J.; Feng, W. Facile synthesis of analogous graphene quantum dots with sp2 hybridized carbon atom dominant structures and their photovoltaic application. Nanoscale 2014, 6, 13043-13052.

(16) Jang, M.-H.; Yang, H.; Chang, Y. H.; Park, H.-C.; Park, H.; Cho, H. H.; Kim, B. J.; Kim, Y.-H.; Cho, Y.-H. Selective engineering of oxygen-containing functional groups using the alkyl ligand oleylamine for revealing the luminescence mechanism of graphene oxide quantum dots. Nanoscale 2017, 9, 18635-18643.

(17) Ye, R.; Xiang, C.; Lin, J.; Peng, Z.; Huang, K.; Yan, Z.; Cook, N. P.; Samuel, E. L. G.; Hwang, C.-C.; Ruan, G.; Ceriotti, G.; Raji, A.-R. O.; Martí, A. A.; Tour, J. M. Coal as an abundant source of graphene quantum dots. Nat. Commun. 2013, 4, 2943.

(18) Peng, J.; Gao, W.; Gupta, B. K.; Liu, Z.; Romero-Aburto, R.; Ge, L.; Song, L.; Alemany, L. B.; Zhan, X.; Gao, G.; Vithayathil, S. A.; Kaipparettu, B. A.; Marti, A. A.; Hayashi, T.; Zhu, J.-J.; Ajayan, P. M. Graphene quantum dots derived from carbon fibers. Nano Lett. 2012, $12,844-849$.

(19) Yuan, J. M.; Zhao, R.; Wu, Z. J.; Li, W.; Yang, X. G. Graphene Oxide Quantum Dots Exfoliated From Carbon Fibers by Microwave Irradiation: Two Photoluminescence Centers and Self-Assembly Behavior. Small 2018, 14, No. e1703714.

(20) Zhang, J.-H.; Sun, T.; Niu, A.; Tang, Y.-M.; Deng, S.; Luo, W.; $\mathrm{Xu}$, Q.; Wei, D.; Pei, D.-S. Perturbation effect of reduced graphene oxide quantum dots (rGOQDs) on aryl hydrocarbon receptor (AhR) pathway in zebrafish. Biomaterials 2017, 133, 49-59.

(21) Pradel, M.; Aissani, L.; Villot, J.; Baudez, J.-C.; Laforest, V. From waste to added value product: towards a paradigm shift in life cycle assessment applied to wastewater sludge - a review. J. Clean. Prod. 2016, 131, 60-75.

(22) Riva, F.; Zuccato, E.; Pacciani, C.; Colombo, A.; Castiglioni, S. A multi-residue analytical method for extraction and analysis of pharmaceuticals and other selected emerging contaminants in sewage sludge. Anal. Methods 2021, 13, 526-535.
(23) Ruiz-Hernando, M.; Cabanillas, E.; Labanda, J.; Llorens, J. Ultrasound, thermal and alkali treatments affect extracellular polymeric substances (EPSs) and improve waste activated sludge dewatering. Process Biochem. 2015, 50, 438-446.

(24) Sheng, G.-P.; Yu, H.-Q.; Li, X.-Y. Extracellular polymeric substances (EPS) of microbial aggregates in biological wastewater treatment systems: a review. Biotechnol. Adv. 2010, 28, 882-894.

(25) Powell, L. C.; Pritchard, M. F.; Ferguson, E. L.; Powell, K. A.; Patel, S. U.; Rye, P. D.; Sakellakou, S.-M.; Buurma, N. J.; Brilliant, C. D.; Copping, J. M.; Menzies, G. E.; Lewis, P. D.; Hill, K. E.; Thomas, D. W. Targeted disruption of the extracellular polymeric network of Pseudomonas aeruginosa biofilms by alginate oligosaccharides. npj Biofilms Microbiomes 2018, 4, 13.

(26) Shi, Y.; Liu, Y. Evolution of extracellular polymeric substances (EPS) in aerobic sludge granulation: Composition, adherence and viscoelastic properties. Chemosphere 2021, 262, 128033.

(27) Ding, Z.; Li, F.; Wen, J.; Wang, X.; Sun, R. Gram-scale synthesis of single-crystalline graphene quantum dots derived from lignin biomass. Green Chem. 2018, 20, 1383-1390.

(28) Thomas, B.; Raj, M. C.; Joy, J.; Moores, A.; Drisko, G. L.; Sanchez, C. Nanocellulose, a Versatile Green Platform: From Biosources to Materials and Their Applications. Chem. Rev. 2018, $118,11575-11625$.

(29) Suryawanshi, A.; Biswal, M.; Mhamane, D.; Gokhale, R.; Patil, S.; Guin, D.; Ogale, S. Large scale synthesis of graphene quantum dots (GQDs) from waste biomass and their use as an efficient and selective photoluminescence on-off-on probe for $\mathrm{Ag}+$ ions. Nanoscale 2014, $6,11664-11670$

(30) Jing, S.; Zhao, Y.; Sun, R.-C.; Zhong, L.; Peng, X. Facile and High-Yield Synthesis of Carbon Quantum Dots from BiomassDerived Carbons at Mild Condition. ACS Sustainable Chem. Eng. 2019, 7, 7833-7843.

(31) Cui, Y.; Liu, R.; Ye, F.; Zhao, S. Single-excitation, dual-emission biomass quantum dots: preparation and application for ratiometric fluorescence imaging of coenzyme A in living cells. Nanoscale 2019, 11, 9270-9275.

(32) Abbas, A.; Mariana, L. T.; Phan, A. N. Biomass-waste derived graphene quantum dots and their applications. Carbon 2018, 140, 77-99.

(33) Wang, Z.; Yu, J.; Zhang, X.; Li, N.; Liu, B.; Li, Y.; Wang, Y.; Wang, W.; Li, Y.; Zhang, L.; Dissanayake, S.; Suib, S. L.; Sun, L. Large-Scale and Controllable Synthesis of Graphene Quantum Dots from Rice Husk Biomass: A Comprehensive Utilization Strategy. ACS Appl. Mater. Interfaces 2016, 8, 1434-1439.

(34) Deng, J.; Xiong, T.; Xu, F.; Li, M.; Han, C.; Gong, Y.; Wang, H.; Wang, Y. Inspired by bread leavening: one-pot synthesis of hierarchically porous carbon for supercapacitors. Green Chem. 2015, 17, 4053-4060.

(35) Qin, Y.; Cheng, Y.; Jiang, L.; Jin, X.; Li, M.; Luo, X.; Liao, G.; Wei, T.; Li, Q. Top-down Strategy toward Versatile Graphene Quantum Dots for Organic/Inorganic Hybrid Solar Cells. ACS Sustainable Chem. Eng. 2015, 3, 637-644.

(36) Zhao, Y.; Wu, X.; Sun, S.; Ma, L.; Zhang, L.; Lin, H. A facile and high-efficient approach to yellow emissive graphene quantum dots from graphene oxide. Carbon 2017, 124, 342-347.

(37) Huang, Q.; Lin, X.; Tong, L.; Tong, Q.-X. Graphene Quantum Dots/Multiwalled Carbon Nanotubes Composite-Based Electrochemical Sensor for Detecting Dopamine Release from Living Cells. ACS Sustainable Chem. Eng. 2020, 8, 1644-1650.

(38) Lyu, B.; Li, H.-J.; Xue, F.; Sai, L.; Gui, B.; Qian, D.; Wang, X.; Yang, J. Facile, gram-scale and eco-friendly synthesis of multi-color graphene quantum dots by thermal-driven advanced oxidation process. Chem. Eng. J. 2020, 388, 124285.

(39) Zhang, Y.; Li, K.; Ren, S.; Dang, Y.; Liu, G.; Zhang, R.; Zhang, K.; Long, X.; Jia, K. Coal-Derived Graphene Quantum Dots Produced by Ultrasonic Physical Tailoring and Their Capacity for $\mathrm{Cu}$ (II) Detection. ACS Sustainable Chem. Eng. 2019, 7, 9793-9799.

(40) Wen, J.; Xie, J.; Zhang, H.; Zhang, A.; Liu, Y.; Chen, X.; Li, X. Constructing Multifunctional Metallic Ni Interface Layers in the g- 
C3N4 Nanosheets/Amorphous NiS Heterojunctions for Efficient Photocatalytic H2 Generation. ACS Appl. Mater. Interfaces 2017, 9, 14031-14042.

(41) Konwar, A.; Baruah, U.; Deka, M. J.; Hussain, A. A.; Haque, S. R.; Pal, A. R.; Chowdhury, D. Tea-Carbon Dots-Reduced Graphene Oxide: An Efficient Conducting Coating Material for Fabrication of an E-Textile. ACS Sustainable Chem. Eng. 2017, 5, 11645-11651.

(42) Kumawat, M. K.; Thakur, M.; Gurung, R. B.; Srivastava, R. Graphene Quantum Dots from Mangifera indica: Application in Near-Infrared Bioimaging and Intracellular Nanothermometry. ACS Sustainable Chem. Eng. 2017, 5, 1382-1391.

(43) Wu, H.; Geng, J.; Ge, H.; Guo, Z.; Wang, Y.; Zheng, G. EggDerived Mesoporous Carbon Microspheres as Bifunctional Oxygen Evolution and Oxygen Reduction Electrocatalysts. Adv. Energy Mater. 2016, 6, 1600794.

(44) Dassanayake, A. C.; Wickramaratne, N. P.; Hossain, M. A.; Perera, V. S.; Jeskey, J.; Huang, S. D.; Shen, H.; Jaroniec, M. Prussian blue-assisted one-pot synthesis of nitrogen-doped mesoporous graphitic carbon spheres for supercapacitors. J. Mater. Chem. A 2019, 7, 22092-22102.

(45) Di, T.; Zhu, B.; Cheng, B.; Yu, J.; Xu, J. A direct Z-scheme g$\mathrm{C} 3 \mathrm{~N} 4 / \mathrm{SnS} 2$ photocatalyst with superior visible-light $\mathrm{CO} 2$ reduction performance. J. Catal. 2017, 352, 532-541.

(46) Li, Y.; Du, Q.; Liu, T.; Peng, X.; Wang, J.; Sun, J.; Wang, Y.; Wu, S.; Wang, Z.; Xia, Y.; Xia, L. Comparative study of methylene blue dye adsorption onto activated carbon, graphene oxide, and carbon nanotubes. Chem. Eng. Res. Des. 2013, 91, 361-368.

(47) Xia, Y.; Mokaya, R.; Walker, G. S.; Zhu, Y. Superior CO2 Adsorption Capacity on N-doped, High-Surface-Area, Microporous Carbons Templated from Zeolite. Adv. Energy Mater. 2011, 1, 678683.

(48) Chen, F.; Guo, L.; Zhang, X.; Leong, Z. Y.; Yang, S.; Yang, H. $Y$. Nitrogen-doped graphene oxide for effectively removing boron ions from seawater. Nanoscale 2017, 9, 326-333.

(49) Zhang, F.; Xue, X.; Huang, X.; Yang, H. Adsorption and heterogeneous Fenton catalytic performance for magnetic Fe3O4/ reduced graphene oxide aerogel. J. Mater. Sci. 2020, 55, 1569515708 .

(50) Zheng, W.; Feng, S.; Feng, S.; Ni, Z.; Shao, C. A novel S-doped $\mathrm{PB} / \mathrm{GO}$ nanocomposite for efficient adsorption and removal of cesium ions. J. Radioanal. Nucl. Chem. 2020, 326, 879-891.

(51) Hu, Q.-L.; Wang, L.-S.; Yu, N.-N.; Zhang, Z.-F.; Zheng, X.; Hu, X.-M. Preparation of Fe3O4@C@TiO2 and its application for oxytetracycline hydrochloride adsorption. Rare Met. 2020, 39, 13331340.

(52) You, L.; Huang, C.; Lu, F.; Wang, A.; Liu, X.; Zhang, Q. Facile synthesis of high performance porous magnetic chitosan polyethylenimine polymer composite for Congo red removal. Int. J. Biol. Macromol. 2018, 107, 1620-1628.

(53) Guo, X.-Y.; Ma, Z.-C.; Liu, G.-W.; Li, D.; Tian, Q.-H. Adsorption of $\operatorname{Re}(\mathrm{VII})$ by coated solvent-impregnated resins containing Alamine 304-1 from sulfuric acid solutions. Rare Met. 2020, 39, 942-950.

(54) Li, J.; Li, H.; Yuan, Z.; Fang, J.; Chang, L.; Zhang, H.; Li, C. Role of sulfonation in lignin-based material for adsorption removal of cationic dyes. Int. J. Biol. Macromol. 2019, 135, 1171-1181.

(55) da Silva, L. G.; Ruggiero, R.; Gontijo, P. d. M.; Pinto, R. B.; Royer, B.; Lima, E. C.; Fernandes, T. H. M.; Calvete, T. Adsorption of Brilliant Red 2BE dye from water solutions by a chemically modified sugarcane bagasse lignin. Chem. Eng. J. 2011, 168, 620-628.

(56) Dong, Y.; Wang, R.; Li, H.; Shao, J.; Chi, Y.; Lin, X.; Chen, G. Polyamine-functionalized carbon quantum dots for chemical sensing. Carbon 2012, 50, 2810-2815.

(57) Brown, M. J.; Lester, J. N. Comparison of bacterial extracellular polymer extraction methods. Appl. Environ. Microbiol. 1980, 40, 179185.

(58) Liu, G.; Niu, P.; Sun, C.; Smith, S. C.; Chen, Z.; Lu, G. Q.; Cheng, H.-M. Unique Electronic Structure Induced High Photo- reactivity of Sulfur-Doped Graphitic C3N4. J. Am. Chem. Soc. 2010, 132, 11642-11648.

(59) Goettmann, F.; Fischer, A.; Antonietti, M.; Thomas, A. Chemical Synthesis of Mesoporous Carbon Nitrides Using Hard Templates and Their Use as a Metal-Free Catalyst for Friedel-Crafts Reaction of Benzene. Angew. Chem., Int. Ed. 2006, 45, 4467-4471.

(60) Ong, W.-J.; Tan, L.-L.; Ng, Y. H.; Yong, S.-T.; Chai, S.-P. Graphitic Carbon Nitride (g-C3N4)-Based Photocatalysts for Artificial Photosynthesis and Environmental Remediation: Are We a Step Closer To Achieving Sustainability? Chem. Rev. 2016, 116, 7159-7329.

(61) Ye, D.; Wang, L.; Zhang, R.; Liu, B.; Wang, Y.; Kong, J. Facile preparation of $\mathrm{N}$-doped mesocellular graphene foam from sludge flocs for highly efficient oxygen reduction reaction. J. Mater. Chem. A 2015, 3, $15171-15176$ 